\title{
Reduction of Qtc interval dispersion. Patential mechanism of cardiac protection of pyridostigmine bromide
}

\author{
Renata Rodrigues Teixeira de Castro, Salvador Manoel Serra, A ntonio Claudio Lucas da Nóbrega
}

\author{
Niterói, RJ - Brazil
}

\begin{abstract}
Objective - Parasympathetic dysfunction is an independent risk factor in individuals with coronary artery disease, and cholinergic stimulation is a potential therapeutical option. We determined the effects of pyridostigmine bromide, a reversible anticholinesterase agent, on electrocardiographic variables of healthy individuals.
\end{abstract}

Methods - We carried out a cross-sectional, double blind, randomized, placebo-controlled study. We obtained electrocardiographic tracings in 12 simultaneous leads of 10 healthy young individuals at rest before and after oral administration of $45 \mathrm{mg}$ of pyridostigmine or placebo.

Results - Pyridostigmine increased RR intervals (before: $886 \pm 27 \mathrm{~ms}$ vs after: $1054 \pm 37 \mathrm{~ms}$ ) and decreased QTc dispersion (before: $72 \pm 9 m s$ vs after: $45 \pm 3 m s$ ), without changing other electrocardiographic variables (PR segment, QT interval, QTc, and QT dispersion).

Conclusion - Bradycardia and the reduction in QTC dispersion induced by pyridostigmine may effectively represent a protective mechanism if these results can be reproduced in individuals with cardiovascular diseases.

Key words- autonomic nervous system, QTc dispersion, pyridostigmine

\footnotetext{
Universidade Federal Fluminense - Niterói e Hospital Pró-Cardíaco - Rio de Janeiro Mailing address: Antonio Claudio Lucas da Nóbrega - Depto de Fisiologia Instituto Biomédico-UFF - Rua Cinco de Julho, 318/1001 - 24220-111 - Niterói, RJ, Brazil

English version by Syela Maris C. Gandour
}

Cardiovascular diseases are one of the major health problems in the industrialized world ${ }^{1}$, including Brazil ${ }^{2}$. In the United States of America, 1.5 million people experience annually acute myocardial infarction, i.e., every 20 s one person experiences acute myocardial infarction ${ }^{1}$. Therefore, it is increasingly necessary to identify and control not only the risk factors for developing the atherosclerotic process, but also the conditions that increase mortality in patients with heart disease, and acute myocardial infarction.

Hypoactivity of the parasympathetic branch of the autonomic nervous system (ANS), identified by a reduction in the heart rate variability, is an independent risk factor in patients after acute myocardial infarction ${ }^{3,4}$. Odemuyiwa et $\mathrm{al}^{5}$ reported that the low heart rate variability, when compared with the reduced left ventricular ejection fraction, has a similar predictive power for overall mortality. On the other hand, the low heart rate variability proved to be a better predictor of sudden death and arrhythmic events than the ejection fraction in the first 6 months after acute myocardial infarction ${ }^{5}$.

Even though recognizing the clinical importance of sympathetic hyperactivity and its treatment in cardiovascular diseases ${ }^{6}$, studies evaluating therapeutical alternatives for low vagal activity are scarce. Pyridostigmine bromide is an anticholinesterase agent that causes a parasympathomimetic effect by increasing the concentration of endogenous acetylcholine. This drug is widely used in patients with myasthenia gravis to increase the concentration of acetylcholine in the motor plate and, therefore, reduce the deficit in muscular strength.

We have currently developed a research line aiming to assess in a systematic way the effects of administration of pyridostigmine bromide on the cardiovascular system and its potential role in correcting vagal hypoactivity. The specific objective of the present study was to investigate the effects of administering pyridostigmine bromide on the electrocardiographic variables of healthy young individuals at rest. 


\section{Methods}

Ten healthy volunteers ( 3 males and 7 females) with a mean age (mean \pm standard deviation) of $28 \pm 6$ years, weighing $67.4 \pm 16.5 \mathrm{~kg}$, and measuring $170 \pm 6 \mathrm{~cm}$ of height underwent a cross-sectional, double-blind, randomized protocol on two different mornings. After resting in the dorsal decubitus position, a surface electrocardiogram with 12 simultaneous leads (software ErgoPC $®$, Micromed, Brazil) was performed on each volunteer, before and 2 hours after oral administration of $45 \mathrm{mg}$ of pyridostigmine bromide (Mestinon ${ }^{\circledR}$, Roche, Brazil) or placebo. All individuals were nonsmokers in a fasting period and were not on any medication. They were instructed not to ingest any substance containing alcohol or caffeine and to avoid strenuous physical exercise during the 2 days preceding the examination. These individuals were considered healthy based on normal results of clinical and electrocardiographic examinations, twodimensional echocardiography with Doppler, and cardiopulmonary exercise testing.

All volunteers gave written consent to take part in the study after being informed about the procedures they would undergo and risks they would be exposed to. The study was approved by the Institutional Committee on Ethics.

The same observer measured the following variables in the 12 electrocardiographic leads: PR segment, RR and QT intervals. In addition, QTc interval, and QT and QTe dispersions were calculated. The software used recorded the 12 electrocardiographic leads simultaneously in a digital form, storing the signals for later analysis. During the analysis, tracings and time recording could be widened, allowing measurements with greater resolution. To compare the values calculated, we used the arithmetic mean of the results obtained in the 12 leads. The simultaneous 12-lead recording is an indispensable tool for calculating spatial dispersion of QT and QTc intervals at the same time.

The QT interval was measured from the first deflection of the QRS complex until the return point of the $T$ wave to the base line or the lowest point between the $\mathrm{T}$ and $\mathrm{U}$ waves? Correction of the QT interval for the heart rate $(\mathrm{QTc})$ was obtained through Bazett's formula $(\mathrm{QT} / \sqrt{\mathrm{RR}})^{8-10}$. Dispersions of the QT and QTc intervals were calculated, respectively, as the subtraction between the greatest and the smallest QT and QTc intervals in the 12 electrocardiographic leads ${ }^{11-13}$.

Statistical assessment of data was based on a repeated measurements analysis of variance (ANOVA). When the $\mathrm{F}$ value was significant, the ANOVA was followed by the Student-Newman-Keuls test for post-hoc paired comparisons. For the statistical analysis of the symptoms reported by the patients while using pyridostig-mine and placebo, we used the chi-square test. The results were considered statistically significant when $\mathrm{p}<0.05$.

\section{Results}

Even though undesired effects occurred with a greater frequency with the use of pyridostigmine $(\mathrm{p}=0.041)$, the symptoms were mild, self-limited, and were reported by only 4 individuals as follows: sialorrhea $(n=3)$, abdominal colic and diarrhea $(\mathrm{n}=1)$, and epigastric discomfort $(\mathrm{n}=1)$.

Two hours after administration of pyridostigmine, we observed significant bradycardia $(\mathrm{p}=0.01)$ (table I) shown as an increase in the duration of the RR intervals and reduction in QTc dispersion. No significant differences were observed in the other electrocardiographic variables studied (QT and QTc intervals, QT dispersion, and PR segment).

\section{Discussion}

Autonomic nervous system dysfunction due to sympathetic hyperactivity or parasympathetic hypoactivity is associated with an increase in the risk of arrhythmias and other cardiac events in patients with heart disease ${ }^{4,14}$. Therefore, dysautonomia increases cardiovascular morbidity and mortality. Adrenergic hyperactivity after acute myocardial infarction has been known for decades, and its clinical relevance has been soundly characterized by a reduction in mortality in patients treated with beta-blockers ${ }^{15}$, a management currently considered standard in the postacute myocardial infarction treatment ${ }^{16}$. More recently, analysis of neuroendocrine features of congestive heart failure has culminated with the indication for use of beta-blockers also in this disease ${ }^{17}$. Even though a reduction in mortality in congestive heart failure has been observed specifically with the use of carvedilol ${ }^{18}$, the general concept that a reduction in the noxious effects of the sympathetic hyperactivity on the heart has been characterized as an efficacious way of reducing cardiac events.

On the other hand, parasympathetic dysfunction gained importance from studies showing its role as an independent risk factor in patients after acute myocardial infarction ${ }^{3,5}$, and, more recently, also in patients with congestive heart failure ${ }^{19}$. Paradoxically, studies aiming to investigate possible specific therapeutical measures for vagal hypoactivity are scarce. Aerobic training may promote an increase in vagal cardiac activity as shown by the greater variability heart rate of patients undergoing this type of training ${ }^{20}$. In regard to possible pharmacological alternatives, in the beginning of the '90s, four independent groups ${ }^{21-24}$ published studies about the cardiovascular effects of scopolamine after acute

\begin{tabular}{|c|c|c|c|c|}
\hline \multicolumn{5}{|c|}{$\begin{array}{l}\text { Table I - Electrocardiographic variables of healthy individuals at } \\
\text { rest before (pre) and } 2 \mathrm{~h} \text { after (post) oral administration of placebo } \\
\text { or } 45 \mathrm{mg} \text { of pyridostigmine in different days }\end{array}$} \\
\hline \multirow[t]{2}{*}{ Variable (ms) } & \multicolumn{2}{|c|}{ Placebo } & \multicolumn{2}{|c|}{ Pyridostigmine } \\
\hline & Pre & Post & Pre & Post \\
\hline PR Segment & $87 \pm 5$ & $88 \pm 5$ & $80 \pm 5$ & $88 \pm 6$ \\
\hline RR Interval & $913 \pm 35$ & $936 \pm 51$ & $886 \pm 27$ & $1054 \pm 37 *$ \\
\hline QT Interval & $366 \pm 8$ & $368 \pm 9$ & $372 \pm 8$ & $386 \pm 9$ \\
\hline QT Dispersion & $55 \pm 5$ & $54 \pm 7$ & $60 \pm 8$ & $47 \pm 5$ \\
\hline QTc Interval & $387 \pm 7$ & $383 \pm 7$ & $396 \pm 7$ & $377 \pm 6$ \\
\hline QTc Dispersion & $60 \pm 6$ & $60 \pm 7$ & $72 \pm 9$ & $45 \pm 3 *$ \\
\hline
\end{tabular}




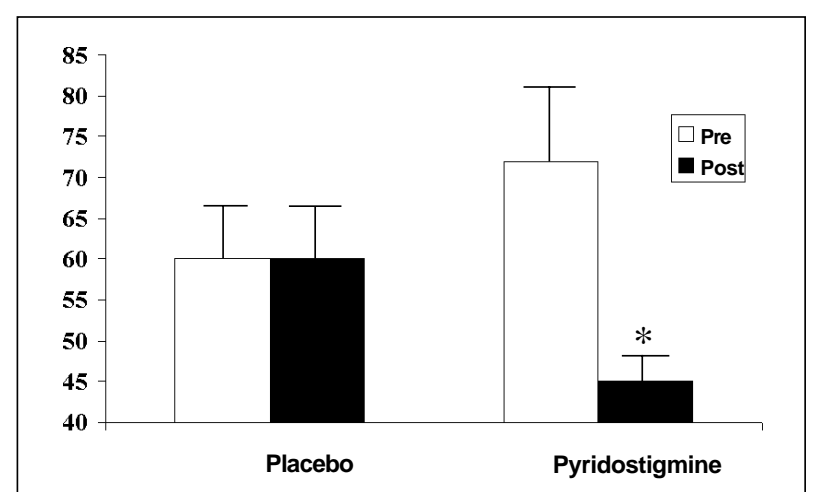

Fig. 1 - Corrected QT interval (QTc) of healthy individuals at rest before (pre) and $2 \mathrm{~h}$ after (post) oral administration of placebo or $45 \mathrm{mg}$ of pyridostigmine in different days $* \mathrm{P}=0.04$ vs pre pyridostigmine.

myocardial infarction. Scopolamine is a muscarinic cholinergic antagonist that may have a parodoxical effect when administered at low doses $(0.4 \text { to } 0.6 \mathrm{mg})^{25}$. All those studies ${ }^{21-24}$ showed a reduction in heart rate and an increase heart rate variability, therefore, suggesting that scopolamine might have a protective effect against cardiac events after acute myocardial infarction. However, the only study that specifically investigated this hypothesis has not confirmed the speculation of the above cited authors ${ }^{26}$. Hull $\mathrm{Jr}$ et al ${ }^{26}$ found that, even though the drug increases heart rate variability in chronically instrumented, dogs it was not able to prevent ventricular fibrillation during physical exertion and artificially induced coronary artery occlusion. Therefore, no experimental or clinical studies exist indicating a pharmacological therapeutical alternative for parasympathetic hypoactivity that may protect against arrhythmogenic sudden death and cardiac mortality in general. Thus, the search for drugs that may cause cholinergic cardiac stimulation and reactivate the possibility of treating vagal hypoactivity present in diverse cardiovascular diseases still continues.

Pyridostigmine bromide is a reversible anticholinesterase agent that does not cross the blood-brain barrier at usual doses and causes a cholinomimetic action by decelerating hydrolysis of endogenous acetylcholine and, consequently, increasing its concentration in the synaptic cleft. Its mostly known clinical indication is for the treatment of the skeletal muscular paralysis of myasthenia gravis ${ }^{27}$, in which the daily dosage may reach $720 \mathrm{mg}$. Pyridostigmine may also be used in multiple sclerosis, amyotrophic lateral sclerosis, spinal myotrophies, and paresis consecutive to poliomyelitis. Other less common indications of pyridostigmine include prevention of disorders after lumbar puncture and meningism after electroencephalography, and treatment of migraine and headache. Another recently reported use ${ }^{28}$ of pyridostigmine was the intake of $30 \mathrm{mg}$ every 8 hours by the American soldiers who took part in the Gulf War. The purpose was to prevent intoxication by gases with irreversible anticholinesterase action in case of an attack with chemical weapons.

The cardiovascular action of pyridostigmine is usually considered a side effect, because in the treatment of myasthenia gravis, the objective is to cause a cholinomimetic effect on the skeletal muscle. This is why systematic studies of the hemodynamic effects of pyridostigmine are not found.

Our team has been studying the cardiovascular action of pyridostigmine. The single dose of $30 \mathrm{mg}$ caused bradycardia at rest ${ }^{29}$ and during dynamic exercise in healthy individuals without, however, impairing tolerance to exertion ${ }^{30}$. In addition, pyridostigmine maintained bradycardia for 24 hours with the use of $30 \mathrm{mg}$ every 8 hours, and it increased in heart rate variability analyzed in the time domain ${ }^{31}$. These effects observed in healthy volunteers occurred with no change in systolic and diastolic cardiac function ${ }^{32}$. When used at the oral dosage of $45 \mathrm{mg}$, pyridostigmine limited the elevation of the double product of healthy individuals under mental stress in a cross-sectional, double blind, randomized, placebo-controlled study ${ }^{33}$.

Parasympathomimetic drugs are known to lower the conduction of the electric cardiac impulse, inducing bradycardia. Drugs that increase duration of the RR intervals on the electrocardiogram would also potentially increase other electrocardiographic variables, such as the PR segment and the QT interval. Depending on the magnitude of these increases, they could mean, respectively, pathological decrease in atrioventricular conduction (atrioventricular blocks of different degrees) or increase in duration of ventricular repolarization. Therefore, when parasympathomimetic drugs are studied, evaluation of their potential effects on clinically relevant electrocardiographic variables are mandatory independently of their effect on heart rate.

Even though some authors consider it unnecessary to calculate the QTc interval, i.e., to correct the QT interval according to heart rate, in our case, this correction is very important. As the drug we are studying is known to cause bradycardia, the calculation of the QTc interval allows a better analysis of cardiac repolarization independent of the duration of the RR interval. That is why an analysis of the degree of spatial dispersion of the refractory periods between different areas of the cardiac surface is obtained eliminating the influence that heart rate exerts on such a variable through the analysis of QTc interval dispersion.

QTc dispersion has a clinical importance similar to that of the QT and QTc intervals. Prolongation of QTc dispersion indicates an increase in the degree of temporal dispersion of the refractory periods between different areas of the cardiac surface, potentiating the occurrence of electric stimulus reentrant phenomena and, consequently, an increase in the risk of ventricular arrhythmias and sudden death ${ }^{34}$. In this way, QTc dispersion proved to be an independent predictor of cardiac and cerebrovascular mortality ${ }^{35-37}$. On the other hand, a smaller QTc dispersion is associated with a smaller mortality rate after acute myocardial infarction ${ }^{38}$, in addition to improving prognosis in individuals with congestive heart failure ${ }^{39}$. In our study, administration of pyridostigmine bromide increased the duration of the RR interval and decreased QTc dispersion without altering the other electrocardiographic variables studied. Thus, in addition to 
inducing bradycardia, pyridostigmine bromide at the dose used showed a potential cardioprotective effect in regard to the occurrence of ventricular arrhythmias and sudden death.

The study here described was carried out in healthy young individuals and, therefore, may not be necessarily reproducible in individuals with cardiovascular diseases. In addition, all studies performed so far by our team have involved the use of a single dose ${ }^{29,30,32,33}$, or a maximum 3 doses ${ }^{31}$ of pyridostigmine bromide. Studies with patients may analyze the effect of pyridostigmine bromide administration in fractionated doses at long-term.
Reduction in QTc interval dispersion after pyridostigmine

If the effects demonstrated so far in healthy individuals may be reproduced in these patients, the performance of controlled studies aiming to evaluate a potential protective effect of pyridostigmine may be justifiable.

\section{Acknowledgements}

We thank CNPq and FAPERJ for financial support and Dr. Washington B. Araújo, Cardiolab - Niterói, RJ for technical support.

\section{References}

1. American Heart Association: Heart and Stroke Facts: 1996 Statistical Supplement. Dallas, American Heart Association, 1996, pp. 1-23.

2. DATASUS. Morbidade Hospitalar do SUS. Ministério da Saúde. http:// www.datasus.gov.br

3. Kleiger RE, Miller JP, Bigger JTJ and The Multicenter Post-Infarction Research Group. Decreased heart rate variability and its association with increased mortality after acute myocardial infarction. Am J Cardiol 1987; 59: 256-62.

4. La Rovere MT, Bigger Jr JT, Marcus FI, Mortara A, Schwartz PJ. Baroreflex sensitivity and heart-rate variability in prediction of total cardiac mortality after myocardial infarction. Lancet 1998; 351: 478-84.

5. Odemuyiwa O, Malik M, Farrel TG, et al. A comparision of the predictive carachteristics of heart rate variability and left ventricular ejection fraction for all-cause mortality, arrhythmic events and sudden death after acute myocardial infarction. Am J Cardiol 1991; 64: 434-9.

6. Corr PB, Gillis RA. Autonomic neural influences on the dysrhythmias resulting from myocardial infarction. Circulation Res 1978; 43: 1.

7. Lepeschkin E, Surawicz B. The measurement of the Q-T interval of the electrocardiogram. Circulation 1952; 6; 378-88.

8. Bazett HC. An anallysis of the time-relations of eletrocardiograms. A.N.E 1997; 02(02): 177-94.

9. Ashman R. The normal duration of the Q-T interval. Am Heart J 1962; 23: 522-34.

10. Simonson E, Cady L, Woodbury M. The normal Q-T interval. Am Heart J 1962; 63: 747-53.

11. Higham PD, Campbell RWF. QT dispersion. Br Heart J 1994; 71: 508-10.

12. Hnatkova K, Malik M, Kautzner J, Gang Y, Camm AJ. Adjustment of QT dispersion assessed from 12 lead eletrocardiograms for different numbers of analysed eletrocardiographic leads: comparision of stability of different methods. Br Heart J 1994; 72: 390-6.

13. Pye M, Quinn AC, Cobbe SM. QT interval dispersion: a non-invasive marker of susceptibility to arrhythmia in patients with sustained ventricular arrhythmias? Br Heart J 1994; 71: 511-14.

14. Schwartz PJ, La Rovere MT, Vanoli E. Autonomic nervous system and sudden death. Experimental basis and clinical observations for post-myocardial infarction risk stratification. Circulation 1992; 85(suppl I): I 77-99.

15. Bigger JT, Coromilas J. How do beta-blockers protect after myocardial infarction? An Intern Med 1984; 101: 256-8.

16. Antman EM, Braunwald E. Acute myocardial infarction. In: Braunwald E. Heart Disease. A Textbook of Cardiovascular Medicine. Philadelphia: WB Saunders, 1997; 1184-288.

17. Barnett DB. Beta-blockers in heart failure: a therapeutic paradox. Lancet 1994; 343: 557-8.

18. Packer M, Bristow MR, Cohn JM, et al. The effect of carvedilol on morbidity and mortality in patients with chronic heart failure. N Engl J Med 1996; 334 : 1349-55.

19. Nolan J, Batin PD, Andrews R, et al. Prospective study of heart rate variability and mortality in chronic heart failure: results of the United Kingdom heart failure evaluation and assessment of risk trial (UK-heart). Circulation 1998; 98: 1510-6.

20. La Rovere MT, Mortara A, Sandrome G, Lombardi F. Autonomic nervous system adaptation to short-term exercise training. Chest 1992; 101: 299s-303s.

21. Casadei B, Pipilis A, Sessa F, Conway J, Sleight P. Low doses of scopolamine in- crease cardiac vagal tone in the acute phase of myocardial infarction. Circulation 1993; 88: 353-7.

22. De Ferrari GM, Mantica M, Vanoli E, Hull SS Jr, Schwartz PJ. Scopolamine increases vagal tone and vagal reflexes in patients after myocardial infarction. J Am Coll Cardiol 1993; 22: 1327-34.

23. Pedretti RF, Colombo E, Braga SS, Carú B. Influence of scopolamine on cardiac sympathovagal interaction after acute myocardial infarction. Am J Cardiol 1993; 72: 384-92.

24. Vybiral T, Glaeser DH, Morris G, et al. Effects of low-dose transdermal scopolamine on heart rate variability in acute myocardial infarction. J Am Coll Cardiol 1993; 22: 1320-36.

25. Brown JH, Taylor P. Muscarinic receptor agonists and antagonists. In: Goodman \& Gilman's. The Pharmacological Basis of Therapeutics. Tennessee: McGrawHill, 1996: 149-151.

26. Hull Jr SH, Vanoli E, Adamsom PB, et al. Do increases in markers of vagal activity imply protection from sudden death? The case of scopolamine. Circulation 1995; 91: 2516-9.

27. Taylor P. Anticholinesterase agents. In: Goodman \& Gilman's. The Pharmacological Basis of Therapeutics. Tennessee: McGraw-Hill, 1996: 161-76.

28. Volans AP. Sarin: guidelines on the management of victims of a nerve gas attack. J Accid Emerg Med 1996; 13: 202-6.

29. Nóbrega ACL, Carvalho ACG, Bastos BG. Resting and reflex heart rate responses during cholinergic stimulation with pyridostigmine in humans. Braz J Med Biol Res 1996; 29: 1461-5.

30. Serra SM, Vivacqua R, Ramalho SHR, Santos KB, Bastos BG, Nóbrega ACL. Cardiopulmonary exercise testing during cholinergic stimulation with pyridostigmine in healthy subjects. J Am Coll Cadiol 1998; 31: 407C.

31. Reis AF, Moraes RS, Bastos BG, Ferlin EL, Ribeiro JP, Nóbrega ACL. Heart rate variability during cholinergic stimulation with piridostigmine in healthy subjects. J Am Coll Cardiol 1998; 31: 407C.

32. Pontes PV, Nóbrega ACL, Mesquita ET, Bastos BG, Carvalho ACG, Romêo LJM. Estudo das variáveis hemodinâmicas e da função cardíaca sistólica e diastólica com emprego de piridostigmina. Arq Bras Cardiol, 1999 (no prelo).

33. Nóbrega ACL, Carvalho ACG, Santos KB, Soares PPS. Cholinergic stimulation with pyridostigmine blunts the cardiac responses to mental stress. Clin Auton Res 1999; 9: 1-6.

34. Ashikaga T. Increased QTc dispersion predicts lethal ventricular arrhythmias complicating coronary angioplasty. Am J Cardiol 1998; 15: 814-6

35. de Bruyne MC, Hoes AW, Kors JA, Hofman A, van Bemmel JH, Grobbee DE. QTc dispersion predicts cardiac mortality in the elderly: the Rotterdam Study. Circulation 1998; 97: 467-72.

36. Sawicki PT, Kiwitt S, Bender R, Berger M. The value of QT interval dispersion for identification of total mortality risk in non-insulin-dependent diabetes mellitus. J Intern Med 1998; 243: 49-56.

37. Batur MK, Aksoyek S, Oto A, Yildirir A, Ozer N, Atalar E, et al. Circadian variations of QTc dispersion: is it a clue to morning increase in sudden cardiac death? Clin Cardiol 1999; 22: 103-6.

38. Glancy JM, Garratt CJ, Woods KL, de Bono DP. Qt dispersion and mortality after myocardial infarction. Lancet 1995; 345: 945-8.

39. Barr CS, Naas AA, Fenwick M, Struthers AD. Enalapril reduces QTc dispersion in mild congestive heart failure secondary to coronary artery disease. Am J Cardiol 1997; 79: 328-33. 